\title{
New insights on glomerular hyperfiltration: a Japanese autopsy study
}

\author{
Go Kanzaki, ${ }^{1,2}$ Victor G. Puelles, ${ }^{1,3}$ Luise A. Cullen-McEwen, ${ }^{1}$ Wendy E. Hoy, ${ }^{4}$ Yusuke Okabayashi, ${ }^{2}$ \\ Nobuo Tsuboi, ${ }^{2}$ Akira Shimizu, ${ }^{5}$ Kate M. Denton, ${ }^{6}$ Michael D. Hughson, ${ }^{7}$ Takashi Yokoo, ${ }^{2}$ \\ and John F. Bertram 1 \\ ${ }^{1}$ Cardiovascular Program, Monash Biomedicine Discovery Institute and Department of Anatomy and Developmental \\ Biology, School of Biomedical Sciences, Monash University, Melbourne, Victoria, Australia. ${ }^{2}$ Division of Nephrology and \\ Hypertension, Department of Internal Medicine, The Jikei University School of Medicine, Tokyo, Japan. ${ }^{3}$ Department of \\ Nephrology and Clinical Immunology, University Hospital RWTH Aachen, Aachen, Germany. ${ }^{4}$ Centre for Chronic Disease, \\ The University of Queensland, Brisbane, Australia. ${ }^{5}$ Department of Analytic Human Pathology, Nippon Medical School, \\ Tokyo, Japan. ${ }^{6}$ Cardiovascular Program, Monash Biomedicine Discovery Institute and Department of Physiology, School \\ of Biomedical Sciences, Monash University, Melbourne, Victoria, Australia. ${ }^{7}$ Department of Pathology, University of \\ Mississippi Medical Center, Jackson, Mississippi, USA.
}

It has been suggested that low nephron number contributes to glomerular hypertension and hyperperfusion injury in progressive chronic kidney disease (CKD). The incidence of CKD in Japan is among the highest in the world, but the reasons remain unclear. We estimated total nephron (glomerular) number ( $\mathrm{Nglom}_{\mathrm{TOTAL}}$ ) as well as numbers of nonsclerosed ( $\mathrm{Nglom}_{\mathrm{NSC}}$ ) and globally sclerosed glomeruli $\left(\right.$ Nglom $\left._{\mathrm{CsC}}\right)$, and the mean volume of nonsclerosed glomeruli $\left(\right.$ Vglom $\left._{\mathrm{NSC}}\right)$ in Japanese normotensive, hypertensive, and CKD subjects and investigated associations between these parameters and estimated glomerular filtration rate (eGFR). Autopsy kidneys from age-matched Japanese men (9 normotensives, 9 hypertensives, 9 CKD) had nephron number and Vglom ${ }_{\mathrm{NSC}}$ estimated using disector/fractionator stereology. Subject eGFR, single-nephron eGFR (SNeGFR), and the ratio SNeGFR/Vglom ${ }_{\mathrm{NSC}}$ were calculated. Nglom $_{\mathrm{NSC}}$ in Japanese with hypertension $(392,108 \pm 87,605 ; P<0.001)$ and CKD $(268,043 \pm 106,968 ; P<0.001)$ was less than in normotensives $(640,399 \pm 160,016)$. eGFR was directly correlated with $\mathrm{Nglom}_{\mathrm{NSG}}(r=0.70, P<0.001)$ and inversely correlated with Vglom $_{\mathrm{NSC}}(r=-0.53, P<0.01)$. SNeGFR was higher in hypertensives than normotensives $(P=0.03)$, but was similar in normotensives and CKD, while the ratio SNeGFR/Vglom ${ }_{\mathrm{NSC}}$ was similar in normotensives and hypertensives but markedly reduced in CKD. Nephron number in Japanese with hypertension or CKD was low. This results in a higher SNeGFR in hypertensives compared with normotensive and CKD subjects, but lowered SNeGFR/Vglom ${ }_{\mathrm{NSC}}$ in CKD subjects, suggesting that changes in GFR are accommodated by glomerular hypertrophy rather than glomerular hypertension. These findings suggest glomerular hypertrophy is a dominant factor in maintenance of GFR under conditions of low nephron number.

Authorship note: G. Kanzaki and V.G. Puelles are co-first authors.

Conflict of interest: The authors have declared that no conflict of interest exists.

License: This work is licensed under the Creative Commons Attribution 4.0 International License. To view a copy of this license, visit http:// creativecommons.org/licenses/by/4.0/

Submitted: April 3, 2017

Accepted: August 24, 2017

Published: October 5, 2017

Reference information: JCI Insight. 2017;2(19):e94334. https://doi.org/10.1172/jci. insight. 94334 .

\section{Introduction}

Over the past 25 to 30 years, much interest has focused on the relationship between nephron number, adult hypertension, and risk of chronic kidney disease (CKD) (1-3). An association between low nephron number and hypertension has been described in some autopsy studies. Keller et al. (4) reported that 10 German hypertensives had approximately half the number of nephrons as 10 normotensives. In a US cross-sectional community adult autopsy study of 171 African Americans and 131 whites, a weak association was found between low nephron number and hypertension for white females but with no significant relationship for white males or African American males or females $(5,6)$. Australian Aborigines have high rates of endstage renal disease (ESRD) and have notably fewer nephrons than Australian whites, and hypertensive Aboriginals have 30\% fewer nephrons than those without hypertension (7).

No previous study has reported nephron number in an Asian population, despite the fact that Japan competes with Taiwan as having the highest incidence of ESRD in the world, with the Japanese in 2012 reporting an annual rate of 2,365 persons per million (8). Tsuboi et al. (9) showed that low glomerular den- 
sity in Japanese renal biopsies was associated with more rapid progression of primary glomerulonephritis. Based on these findings, Tsuboi et al. proposed that glomerular density, at least in part, reflects the nephron number of each individual and that Japanese subjects may have a low nephron number that potentially marks their risk of hypertension and CKD (10-12).

Autopsy studies have also reported strong inverse correlations between total glomerular number and mean glomerular volume $(5,13,14)$. These findings suggest that larger glomeruli have undergone compensatory hypertrophic growth that tends to normalize the total glomerular volume in a kidney (15). Glomerular hypertrophy also occurs with hypertension and obesity, suggesting that increased metabolic and excretory demands can contribute to compensatory glomerular growth (16-18). Although the effect of increased glomerular volume on glomerular function is unclear (18-21), Denic et al. recently found that single-nephron glomerular filtration rate (SNGFR; measured GFR divided by the number of nephrons) in healthy adult kidney donors was fairly constant with regard to age, sex, and height (if < $190 \mathrm{~cm})(22)$. However, these authors found that higher SNGFR was independently associated with larger nephrons on biopsy and more glomerulosclerosis and arteriosclerosis than would be expected for the donors' age. Because of the close correlation between glomerular number and volume, associations between glomerular volume and SNGFR in the context of hypertensive or CKD status are of particular interest, and may provide new insights into glomerular hyperfiltration.

In the present study, we assessed nephron number at autopsy in 3 age-matched Japanese groups (normotensive, hypertensive, and CKD subjects). We analyzed associations between renal function (estimated GFR, eGFR) and glomerular number and volume in order to investigate the role of single-nephron eGFR (SNeGFR) in the development of hypertension and CKD.

\section{Results}

Demographics. Kidneys from 27 Japanese male subjects were collected at Nippon Medical School (Tokyo, Japan) during autopsy and were divided into 3 age-matched groups: normotensive $(n=9)$, hypertensive $(n$ $=9)$, and CKD $(n=9)$ (Figure 1$)$.

Table 1 provides demographic and clinical data for the normotensive, hypertensive, and CKD subjects. The age range for normotensives was 53 to 72 years, with 2 subjects 70 years or older; for hypertensives was 51 to 84 years, with 5 subjects 70 years or older; and for CKD was 61 to 79 years, with 5 subjects 70 years or older.

Renal function and histopathology. CKD subjects had the lowest eGFR $\left(34.8 \pm 11.8 \mathrm{ml} / \mathrm{min} / 1.73 \mathrm{~m}^{2} ; P<\right.$ 0.001 compared with normotensives and hypertensives). Blood urea nitrogen and uric acid were highest in CKD subjects ( $P=0.04$ and $P=0.03$, respectively). Plasma hemoglobin, total protein, and albumin were similar in the 3 groups, indicating that differences in oncotic pressure were unlikely to influence glomerular hemodynamics. Histopathologic analysis showed that the 9 CKD subjects had benign nephrosclerosis.

Kidney morphometric data and SNeGFR for the 3 groups are presented in Table 2. Kidney weights and cortical volumes were similar in the 3 groups. The extent of glomerulosclerosis, evaluated by calculating a glomerulosclerosis index (GSI; percentage of glomeruli that were sclerotic), was highest in CKD subjects $(P=0.01)$.

Progressive nephron deficit in association with hypertension and $C K D$. Kidneys of hypertensive subjects contained approximately 39\% fewer nonsclerosed glomeruli $\left(\mathrm{Nglom}_{\mathrm{NSG}}\right)$ than those of normotensive subjects $(392,108 \pm 87,605$ [range 264,770-507,077] vs. 640,399 $\pm 160,016$ [range 390,352-938,659], $P<0.001)$. CKD subjects had significantly fewer Nglom $_{\mathrm{NSG}}(268,043 \pm 106,968$ [range 65,086-402,102]) than both hypertensives $(P=0.04)$ and normotensives $(P<0.001)$ (Figure $2 \mathrm{~A})$. Interestingly, despite the GSI being significantly higher in the CKD group than in the other 2 groups, the numbers of globally sclerotic glomeruli $\left(\mathrm{Nglom}_{\mathrm{GSG}}\right)$ in the 3 groups was very similar, and quite low, ranging from 25,741 \pm 9,941 in the normotensive group to $31,390 \pm 19,605$ in the hypertensive group.

Compensatory glomerular hypertrophy in hypertensive and CKD subjects. Compared with normotensives $\left(5.98 \pm 1.44[4.02-7.25] \times 10^{6} \mu \mathrm{m}^{3}\right)$, the mean volume of nonsclerosed glomeruli $\left(V_{\text {glom }}{ }_{\mathrm{NSG}}\right)$ was higher in hypertensives $\left(7.74 \pm 1.43[6.49-9.91] \times 10^{6} \mu \mathrm{m}^{3}, P=0.02\right)$, and markedly higher in CKD subjects $\left(10.86 \pm 3.83\right.$ [6.48-18.92] $\times 10^{6} \mu \mathrm{m}^{3}, P<0.001$ vs. normotensives, $P=0.001$ vs. hypertensives, Figure 2B). Thus, mean glomerular volume in CKD subjects was almost double that in normotensive subjects. There was a strong inverse relationship between $\mathrm{Nglom}_{\mathrm{NSG}}$ and $\mathrm{Vglom}_{\mathrm{NSG}}$, such that $\mathrm{Vglom}_{\mathrm{NSG}_{\text {total }}}$ (the product of $\mathrm{Nglom}_{\mathrm{NSG}}$ and Vglom $\left._{\mathrm{NSG}}\right)$ was similar in the 3 groups $(P=0.06$, Figure $2 \mathrm{C})$. While Nglom $_{\mathrm{NSC}}$ was inversely correlated with glomerulosclerosis $(P<0.01)$, no association was found between $\mathrm{Vglom}_{\mathrm{NSC}}$ and glomerulosclerosis $(P=0.20)$. 


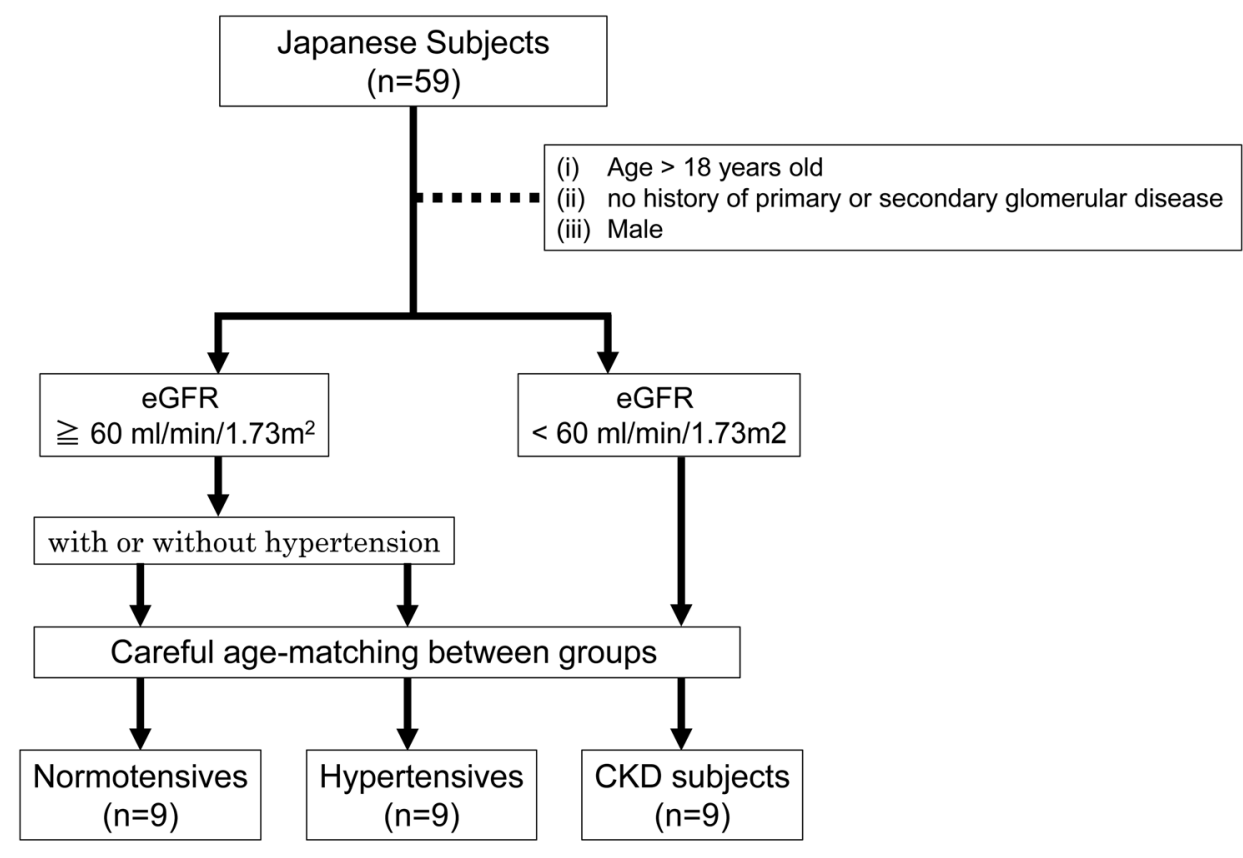

Figure 1. Subject selection. Fifty-nine Japanese kidneys were collected at autopsy. Nine kidneys with CKD were selected according to eGFR less than 60 $\mathrm{ml} / \mathrm{min} / 1.73 \mathrm{~m}^{2}$, and compared with 9 normotensive and 9 hypertensive subjects based on the inclusion criteria. Subjects were age and sex matched. eGFR, estimated glomerular filtration rate; CKD, chronic kidney disease.

Associations between kidney weight, cortical volume, nephron number, and eGFR. Nglom $_{\mathrm{NSG}}$ was strongly and directly associated with eGFR $(r=0.70, P<0.0001$, Figure $3 \mathrm{~A})$, while Vglom ${ }_{\mathrm{NSG}}$ was inversely correlated with eGFR $(r=-0.63, P<0.01$, Figure $3 \mathrm{~B})$. There was a modest correlation between Vglom $_{\mathrm{NS}}$ Gtotal and eGFR $(r=-0.39, P=0.04)$. The association between eGFR and kidney weight did not reach statistical significance $(r=0.36, P=0.07$, Figure $3 \mathrm{C}$ ), and cortical volume was mildly correlated with eGFR ( $r=0.39, P=0.04$, Figure 3D). While there were no statistical differences between normotensive, hypertensive, and CKD subjects in kidney weight $(P=0.08)$ or cortical volume $(P=0.47)$ (Table 2$)$, there was a strong positive correlation between these variables $(r=0.68, P<0.001$, Figure $4 \mathrm{~A})$. Both kidney weight and cortical volume were strongly associated with the number of total glomeruli per kidney (Nglom ${ }_{\text {TOTAL }}$ ) (Figure 4, B and C, respectively).

Associations between $e G F R$ and glomerular number and size. In order to further analyze associations between eGFR and glomerular number and size, we calculated SNeGFR and the ratio between SNeGFR and Vglom $_{\mathrm{NSG}}$. SNeGFR was calculated by dividing eGFR by the total number of nonsclerosed glomeruli per subject $\left(\mathrm{Nglom}_{\mathrm{NSG}} \times 2\right.$, where 2 accounts for 2 kidneys). SNeGFR was significantly higher in hypertensives than in normotensives $(P=0.03)$, but similar in normotensive and CKD subjects $(P=0.81$, Figure $5 \mathrm{~A})$. SNeGFR/Vglom ${ }_{\mathrm{NSG}}$ was similar in normotensives and hypertensives, but about $50 \%$ lower in $\mathrm{CKD}$ subjects $(P<0.001$, Figure 5B).

\section{Discussion}

The main findings from this study of aging Japanese were (a) nephron number in normotensive Japanese is lower than in several other races, (b) hypertensive subjects had fewer nephrons than normotensives, (c) nephron number in CKD subjects was significantly lower than in normotensive and hypertensive subjects, (d) eGFR was directly correlated with the number of nonsclerosed glomeruli, (e) SNeGFR in hypertensive subjects was higher than in normotensives and subjects with $\mathrm{CKD}$, and (f) in CKD subjects reduced nephron number was associated with glomerular hypertrophy and a decrease in the ratio SNeGFR/Vglom ${ }_{\mathrm{NSG}}$. Our data suggest that glomerular hypertrophy may be a dominant factor in maintenance of GFR in the setting of a low nephron number.

Nephron number in normotensive Japanese subjects is one of the lowest nephron counts yet reported. Estimates obtained using the disector/fractionator combination in other races are provided in Table 3 . We speculate that 4 factors may explain this low nephron count in normotensive Japanese.

First, it is possible that the low nephron number in Japanese is simply in proportion to their smaller body size. Denic et al. (23) recently reported a direct correlation between height and glomerular number in 1,638 US kidney donors. With smaller height, it is likely that the Japanese subjects had appropriately 
Table 1. Demographic and clinical data for Japanese subjects

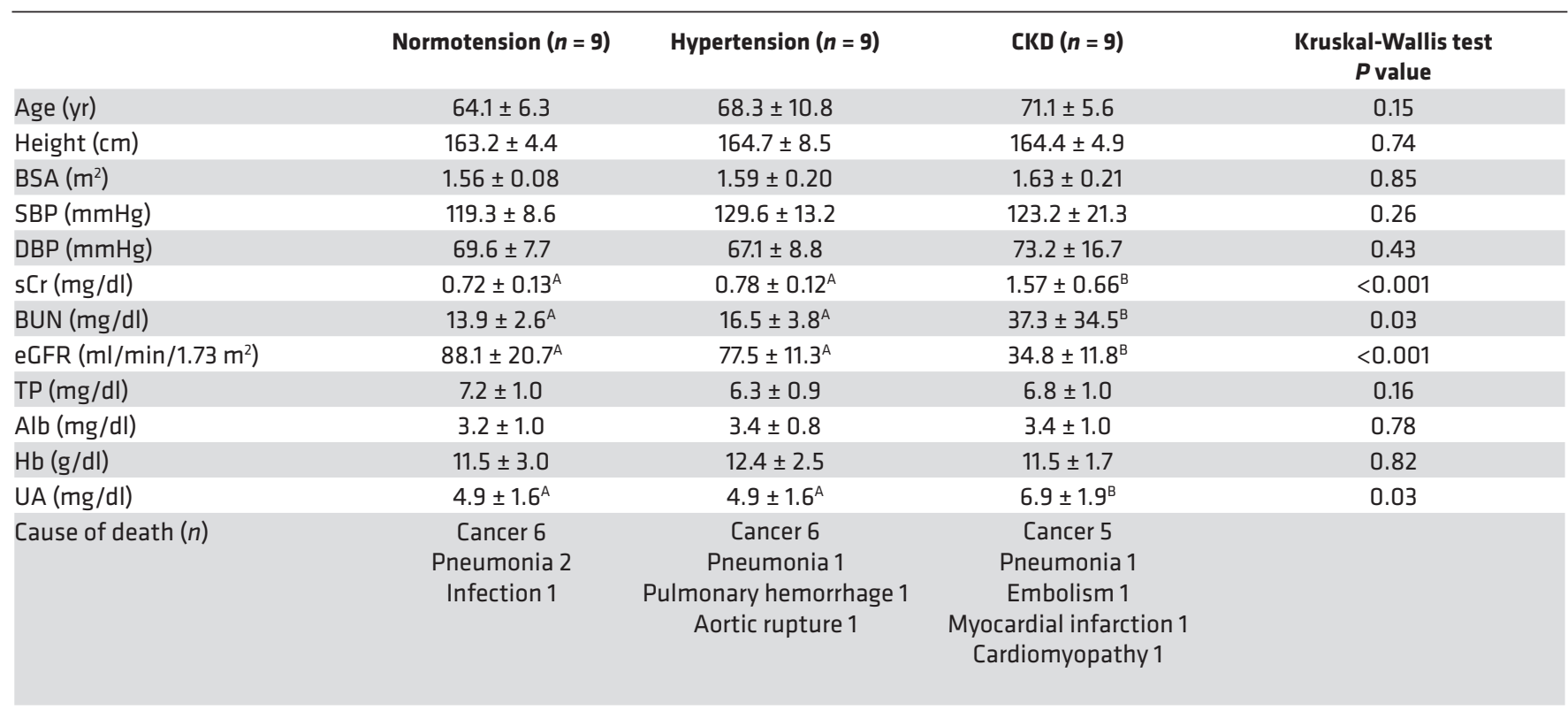

Data analyzed using Kruskal-Wallis test with post-hoc Mann-Whitney $U$ tests with the Bonferroni correction. Values labeled with the same letter are not significantly different, whereas those labeled with a different letter are significantly different. BSA, body surface area; SBP, systolic blood pressure; DBP, diastolic blood pressure; $\mathrm{SCr}$, serum creatinine concentration; BUN, blood urea nitrogen concentration; eGFR, estimated glomerular filtration rate; TP, total protein concentration; Alb, albumin concentration; $\mathrm{Hb}$, hemoglobin concentration; UA, uric acid concentration.

smaller organ size generally and nephron endowment in particular. Second, the low nephron count in Japanese may be related to genetic factors. The Japanese population is considered to have a high level of genetic homogeneity due to centuries of isolation. Several studies have reported genetic variants associated with small human kidney size and low nephron number $(24,25)$.

Third, the Japanese subjects in the present study were born during and shortly after World War II, a time of poverty and poor living conditions in Japan (26). Animal studies have shown that nephron number can be influenced by an adverse feto-maternal environment, and a developmental contribution to adult hypertension and CKD in humans is becoming increasingly accepted $(27,28)$. We previously reported a direct correlation between total nephron number and birth weight in white and African Americans (14). The Dutch Winter Famine (29) and the Leningrad Siege (30), periods of starvation, poverty, and stress, have been linked with adverse health outcomes in adulthood (31-33). It is therefore possible that the Japanese subjects investigated in the present study were born with low birth weight and low nephron endowment. Unfortunately, birth weight data were not available for the Japanese subjects.

Finally, another potential contributor to the low nephron count in normotensive Japanese is nephron loss associated with glomerulosclerosis. In the present study, nephron number was strongly and inversely correlated with glomerulosclerosis. The GSI in normotensive subjects was $4.1 \%$, with a mean number of 25,741 globally sclerotic glomeruli. The average age of normotensive subjects was 64.1 years. Denic et al. (23) recently reported that aging (>70 years) US transplant donors have nearly $50 \%$ fewer nonsclerosed glomeruli than 18- to 40-year-old donors, and this nephron loss is the result of nephrosclerosis with global glomerulosclerosis. Old, obsolescent glomeruli are thought to be absorbed without trace from the kidney, such that the extent of glomerular loss is unaccounted for by the amount of glomerulosclerosis $(23,34)$. Given that 2 of the 9 normotensive subjects were older than 70 years, it is possible that our low nephron counts in normotensive Japanese may also be explained by age-related nephron loss.

Primary hypertension is physiologically and pathologically complex $(35,36)$. Less than $10 \%$ of individuals develop hypertension before 30 years of age. Afterward, hypertension begins to increase in frequency and is reported in more than $60 \%$ of Japanese and US blacks after 65 years of age $(37,38)$. In a sample of German road accident victims, Keller et al. (4) found 50\% fewer glomeruli in 10 hypertensive 
Table 2. Kidney morphometric data and SNeGFR for Japanese subjects

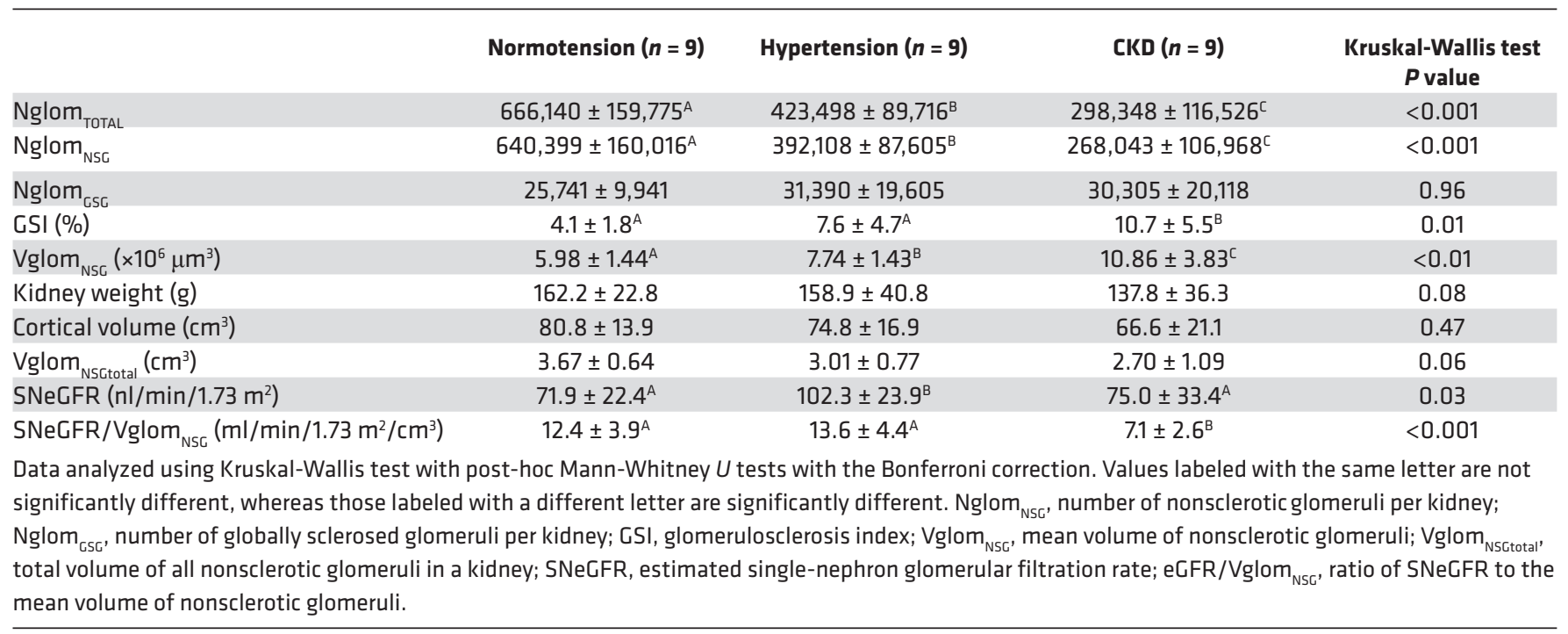

German subjects with a median age of 45 years compared with 10 age-matched normotensive subjects. In contrast, in 14 US subjects with onset of hypertension before 40 years of age, no significant difference in nephron number was found compared with cross-sectional normotensive controls, and it was presumed that nephron number was not related to blood pressure (6). In the present study, 9 Japanese hypertensive subjects with a mean age of 68 years had 37\% fewer nephrons than 9 normotensive subjects with a mean age of 64 years. It remains an unanswered question as to whether the hypertension is primary or secondary to glomerulosclerosis and acquired nephron deficit.

We found that with lower nephron number, SNeGFR was elevated in hypertensive compared with normotensive subjects. Moreover, the Vglom $_{\mathrm{NSG}}$ in $\mathrm{CKD}$ was almost twice that of normotensive kidneys. The lower nephron number in CKD resulted in a markedly decreased eGFR, but with glomerular hypertrophy, SNeGFR was similar to that in normotensive subjects. SNeGFR/Vglom ${ }_{\mathrm{NSG}}$ was significantly lower in CKD subjects than the other 2 groups. Based on these findings, we propose that when nephron number is low, the reduction in eGFR is limited to a large extent by an increase in the renal ultrafiltration coefficient (Kf), associated with hypertrophy of glomeruli and increased glomerular filtration surface area, which enables hyperfiltration.

The associations described above between glomerular number, glomerular volume, and SNeGFR in normotensive, hypertensive, and CKD subjects are somewhat different from those of Denic et al. who measured these parameters in healthy adult kidney donors $(22,23)$. They found that while nephron number decreased with healthy aging, SNGFR remained relatively constant, and glomerular volume did not increase in association with nephron loss (39). They concluded that this failure to compensate for loss of nephrons in healthy aging might be due to a concurrent decrease in metabolic demand that could influence GFR (22).

The glomerular loss occurring in $\mathrm{CKD}$ is mainly the result of global glomerulosclerosis that is also referred to as ischemic glomerular obsolescence $(6,36)$. Hypertension or CKD in the opinion of many in the field is a vascular disease in which glomerular loss is the result of arteriosclerosis in small renal arteries $(36,40,41)$. The very low SNeGFR/Vglom ${ }_{\mathrm{NSG}}$ in CKD indicates that while SNeGFR may be in the normal to high range, there is actually a reduction in glomerular capillary hydrostatic pressure $(\Delta \mathrm{P})$ in the failing nephrons. The preglomerular arteries in CKD subjects in the present study have arteriolosclerosis and therefore increased resistance, which leads to reduced $\triangle \mathrm{P}$ and decreased GFR (42). This would be the anticipated relationship if glomerular obsolescence was initiated by altered perfusion through stenotic preglomerular arteries $(6,36,40,41)$.

In the present study, despite significantly lower nephron numbers in hypertensive and CKD subjects, cortical volume in the 3 groups was similar, suggesting that a significant degree of hypertrophy of remaining nephron tubules had occurred. Low nephron count in humans has previously been associated with tubular hypertrophy (43). In response to the increased filtered load, compensatory cellular hypertrophy in proximal tubules increases $\mathrm{Na}^{+}$reabsorption, which restores $\mathrm{NaCl}$ delivery to distal tubules via 
A

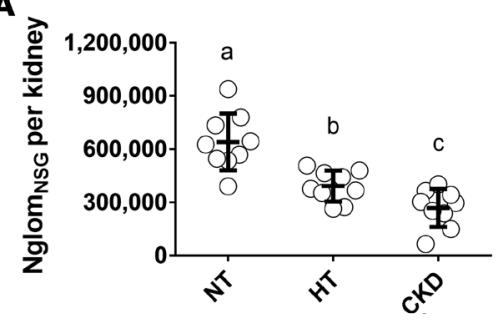

B

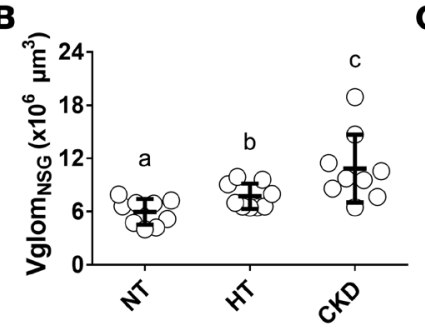

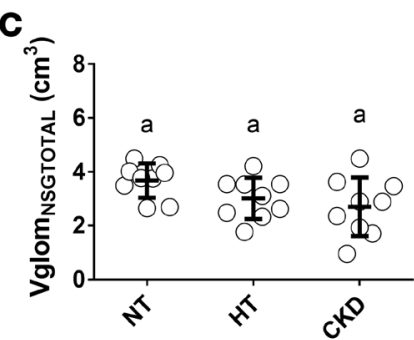

Figure 2. Numbers and volumes of nonsclerosed glomeruli in normotensive, hypertensive, and CKD Japanese subjects. (A) Numbers of nonsclerosed glomeruli $\left(\mathrm{Nglom}_{\mathrm{NSC}}\right.$ ) per kidney, (B) mean volume of nonsclerosed glomeruli $\left(\right.$ Vglom $\left._{\mathrm{NSC}}\right)$, and (C) total volume of all nonsclerosed glomeruli $\left(\right.$ Vglom $\left._{\text {NSCTOTAL }}\right)$ for the 3 Japanese groups. NT, normotensive; HT, hypertensive; CKD, chronic kidney disease. Bold lines indicate means \pm SD. In A-C, $n=9$ each for the NT, $\mathrm{HT}$, and CKD groups. Data were analyzed using Kruskal-Wallis test with post-hoc Mann-Whitney $U$ tests with the Bonferroni correction. Groups labeled with the same letter are similar, whereas those labeled with a different letter are significantly different $(P \leq 0.05)$.

tubuloglomerular feedback (44). We therefore propose that compensatory nephron hypertrophy is sufficient to account for hyperfiltration, as an alternative to the hypothesis of human glomerular hypertensive injury. When perfusion pressure is reduced by increasing arteriosclerosis, the hypertrophied tuft cannot be maintained and collapses with progression to global glomerulosclerosis.

Our study has several limitations. The first is the small number of subjects with the attendant risks of random statistical error. The small sample size was imposed by the need to have age-matched and sex-matched
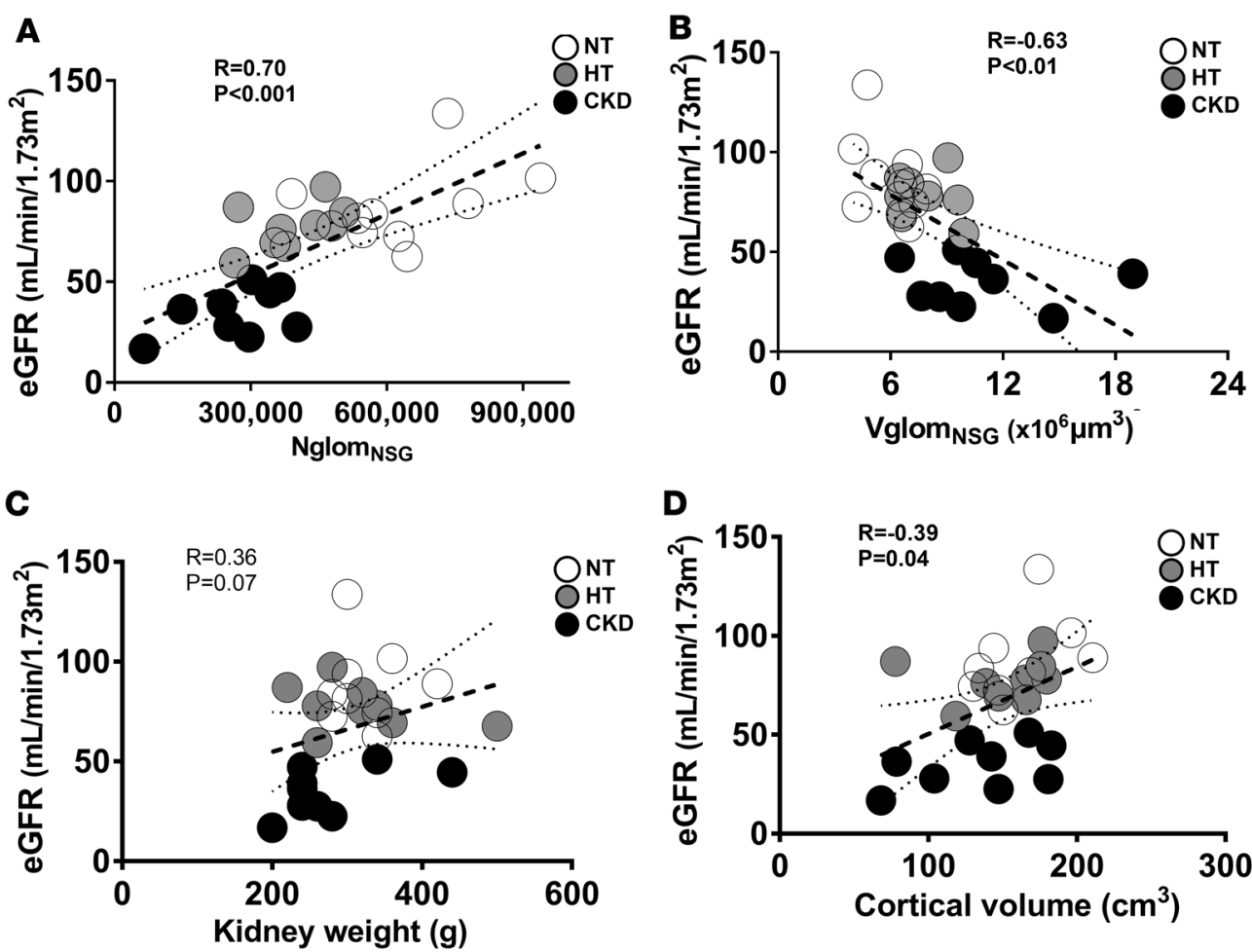

Figure 3. Glomerular number, kidney weight, and cortical volume in relation to eGFR. (A) Associations between estimated glomerular filtration rate (eGFR) and numbers of nonsclerosed glomeruli $\left(\mathrm{Nglom}_{\mathrm{NSC}}\right)$, (B) mean volume of nonsclerosed glomeruli $\left(V_{\text {glom }}\right.$ ) ), (C) kidney weight, and (D) cortical volume. White circles, normotensive (NT); gray circles, hypertensive (HT); black circles, chronic kidney disease (CKD). In A-D, $n=9$ each for the NT, HT, and CKD groups. Measures of association were tested by Spearman rank coefficients. Solid lines are lines of best fit. Dotted lines show $95 \%$ confidence intervals. 
A

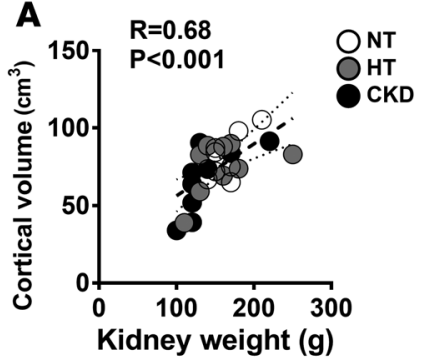

B

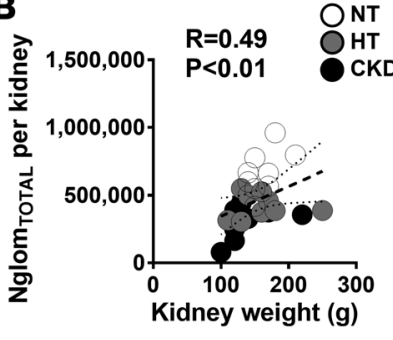

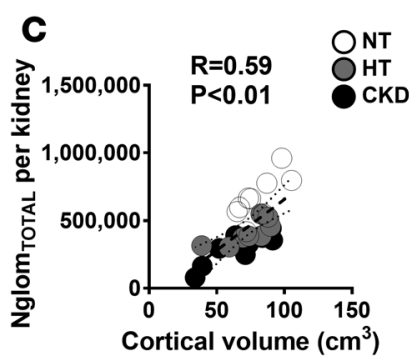

Figure 4. Correlations between kidney weight, cortical volume, and total glomerular number. (A) Associations between kidney weight and cortical volume, (B) kidney weight and total glomerular number (Nglom TOTAL $_{\text {) }}$ per kidney, and (C) cortical volume and Nglom тотаL per kidney. White circles, normotensive (NT); gray circles, hypertensive (HT); black circles, chronic kidney disease (CKD). In A-C, $n=9$ each for the NT, HT, and CKD groups. Measures of association were tested by Spearman rank coefficients. Solid lines are lines of best fit. Dotted lines show $95 \%$ confidence intervals.

subjects. Secondly, morphometric analyses were performed on kidneys obtained at autopsy, while functional data and laboratory data were obtained shortly before death. The death process and the changes occurring after death may have confounded to some extent the relationships between morphometric and clinical/physiological data. In addition, the equation used to estimate GFR may not be totally applicable to the non-CKD subjects. While our estimate of Nglom $_{\text {тОтАL }}$ was obtained using unbiased stereology (disector/fractionator combination), the estimates of $\mathrm{Nglom}_{\mathrm{NSG}}$ and $\mathrm{Nglom}_{\mathrm{GSG}}$ are likely biased because their percentages were based on counts of glomerular profiles in single sections, such that smaller sclerotic glomeruli may have been undersampled compared with larger nonsclerotic glomeruli. However, the numbers of sclerotic glomeruli were small, and we believe the overall bias is similarly small. Moreover, Rosenberg et al. (45) showed that the percentage of sclerotic glomeruli in sections is a reasonable estimate of the percentage of sclerotic glomeruli in the cortex. Our findings may not be applicable to other Asian populations, newer generations of Japanese, or Japanese residents of other countries. Comparative studies would be valuable in younger Japanese as well as US Asians, with the latter in the US Renal Data Survey having no increased risk of hypertensive CKD.

In conclusion, the present findings suggest that lower nephron number in elderly Japanese may be a feature of their susceptibility to hypertensive CKD. We show that eGFR is directly correlated with Nglom $_{\mathrm{NSG}}$, and our findings suggest that maintenance of filtration is principally accommodated by compensatory glomerular hypertrophy rather than glomerular hypertension.

\section{Methods}

Subject selection and kidney collection. Kidneys from 59 Japanese subjects aged 28 to 85 years were collected at Nippon Medical School, Tokyo, Japan, during autopsies performed between January 2010 and November 2014.

Total nephron (glomerular) number $\left(\mathrm{Nglom}_{\mathrm{TOTAL}}\right)$, the numbers of nonsclerotic $\left(\mathrm{Nglom}_{\mathrm{NSG}}\right)$ and globally sclerotic $\left(\right.$ Nglom $\left._{\mathrm{GSG}}\right)$ glomeruli, and the mean volume of NSG (Vglom $\left.{ }_{\mathrm{NSG}}\right)$ were determined in normotensive, hypertensive, and CKD Japanese subjects. Inclusion criteria were (a) death after the age of 18 years, (b) no history of primary or secondary glomerular disease, and (c) male sex. Subjects were categorized into hypertensive and nonhypertensive on the basis of a history of hypertension, consistently elevated blood pressure ( $\geq 140 / 90 \mathrm{mmHg}$ ), or treatment with antihypertensive medications. Subjects with CKD were defined as those with eGFR less than $60 \mathrm{ml} / \mathrm{min} / 1.73 \mathrm{~m}^{2}$. Twenty-seven kidneys were selected based on these criteria and categorized based on hypertensive or CKD status ( $n=9$ normotensives, $n=9$ hypertensives, $n=9$ CKD subjects). eGFR was available within 1 month of death.

Demographic data and clinical variables. General demographic data including age, race, sex, height, body weight, kidney weight, medical history, treatment, blood pressure, and cause of death were obtained from autopsy reports and medical records. Serum laboratory values for creatinine ( $\mathrm{sCr}$ ), blood urea nitrogen, uric acid, hemoglobin, total protein, and albumin were obtained within 1 month before death.

Definitions. eGFR for Japanese subjects was calculated using a modified 3-variable equation of Matsuo et al. (46): eGFR $=194 \times$ Age $^{-0.287} \times \mathrm{sCr}^{-1.094}$. Body surface area (BSA) of Japanese subjects was determined using the equation of Fujimoto et al. (47): BSA $\left(\mathrm{m}^{2}\right)=\operatorname{Weight}^{0.444}(\mathrm{~kg}) \times \operatorname{Height}^{0.663}(\mathrm{~cm}) \times 88.83 \times 10^{-4}$.

Vglom $_{\mathrm{NSG} \text { total }}$ is the product of $\mathrm{Nglom}_{\mathrm{NSG}}$ and Vglom $_{\mathrm{NSG}}$. Glomerular filtration was assessed using 2 parameters: (a) SNeGFR = eGFR/ $\left(2 \times\right.$ Nglom $\left._{\mathrm{NSG}}\right)$, where 2 accounts for 2 kidneys; and (b) SNeGFR/Vglom NSG $^{*}$ 

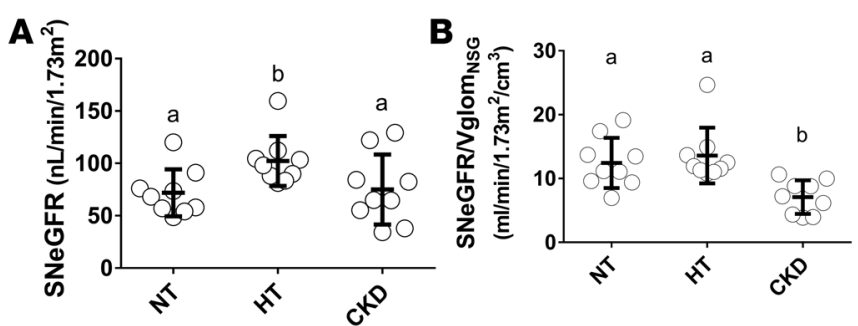

Figure 5. SNeGFR and SNeGFR/Vglom ${ }_{\mathrm{NSG}}$ in normotensive, hypertensive, and CKD Japanese subjects. (A) Estimated single-nephron glomerular filtration rate (SNeGFR) is the ratio of eGFR to twice the number of nonsclerosed glomeruli $\left(\mathrm{Nglom}_{\mathrm{NSC}}\right.$ ). (B) SNeGFR/Vglom $\mathrm{NSC}_{\text {is }}$ is the ratio of SNeGFR to the mean volume of nonsclerosed glomeruli $\left(\mathrm{Vglom}_{\mathrm{NSC}}\right)$. NT, normotensive; HT, hypertensive; CKD, chronic kidney disease. Bold lines indicate means \pm SD. In $\mathbf{A}$ and $\mathbf{B}, n=9$ each for the NT, HT, and CKD groups. Data were analyzed using Kruskal-Wallis test with post-hoc Mann-Whitney $U$ tests with the Bonferroni correction. Groups labeled with the same letter are similar, whereas those labeled with a different letter are significantly different $(P \leq 0.05)$.

Estimation of nephron numbers and mean glomerular volume. To calculate $\mathrm{Nglom}_{\mathrm{TO} \text { (AL }}$, as well as the numbers of $\mathrm{Nglom}_{\mathrm{NSG}}$ and $\mathrm{Nglom}_{\mathrm{GSG}}$, we first estimated $\mathrm{Nglom}_{\mathrm{TOTAL}}$ using the physical disector/fractionator combination at Monash University. Details of this method have been published previously $(48,49)$, but are briefly described here. Kidneys were perfusion-fixed with $10 \%$ formalin, and a known weight fraction of tissue was sampled (fraction 1). Fraction 1 was sliced into approximately $1 \mathrm{~cm} \times 1 \mathrm{~cm} \times 1 \mathrm{~mm}$ tissue blocks and systematically sampled to obtain a slice fraction (fraction 2) and processed for embedding in glycolmethacrylate. Tissue shrinkage in glycolmethacrylate is much less than in paraffin and thereby provides more realistic estimates of glomerular size. These sampled blocks were exhaustively sectioned at $20 \mu \mathrm{m}$, and every 10th and 11th section pair (fraction 3) was stained with Periodic acid-Schiff stain (PAS). Section pairs with complete kidney sections were viewed using paired microscopes fitted with projection arms, and a motorized stage was used to obtain a systematic uniform random sample of microscopic fields for glomerular counting. Nglom ${ }_{\text {TотаL }}$ was estimated using the following equation: $\operatorname{Nglom}_{\text {TOTAL }}=f_{1} \times f_{2} \times f_{3} \times f a \times$ $Q^{-}$, where $f_{1}$ is the weight fraction of tissue sampled (kidney weight/weight of 4-mm slices), $f_{2}$ is the inverse of the slice sampling fraction, $f_{3}$ is the inverse of the section sampling fraction (i.e., $1 / 10$ or 10 ), and $F a=[P s$ $\times a(p)] /[2 \times P f \times a(p)] . P s \times a(p)$ is the sum of points overlying all kidney sections on microfiche multiplied by the area associated with each grid point, and $P f \times a(p)$ is the sum of points overlying complete kidney sections on physical disector multiplied by the area associated with each grid point (13). Glomeruli were counted using the disector principle, in that only those glomeruli present in one section field but absent from the next section in the pair were counted; $Q^{-}$is the number of glomeruli actually counted with the disector. In this study, the average $Q^{-}$per kidney was 117, with values ranging from 82 to 222 .

Because of the low numbers of sclerotic glomeruli in the 3 groups of kidneys, it was not possible to estimate numbers of sclerotic glomeruli using the disector principle, where a $Q^{-}$of around 100 is required to obtain estimates with a suitably low coefficient of error. Therefore, to obtain separate estimates for Nglom $_{\mathrm{NSG}}$ and Nglom $_{\mathrm{GSG}}$ per kidney, profiles of nonsclerotic and sclerotic glomeruli were counted in 1 PAS-stained glycolmethacrylate section from each sampled block per kidney using unbiased counting frames (50). The percentage of nonsclerotic and sclerotic glomerular profiles was calculated for each kidney and then multiplied by Nglom $_{\text {TOTAL }}$ to obtain Nglom $_{\mathrm{NSG}}$ and Nglom $_{\mathrm{GSG}}$.

To estimate the Vglom $_{\mathrm{NSG}}$, the volume density of nonsclerosed glomeruli in renal cortex $\left(\mathrm{V}_{\mathrm{VNSG}, \text { Cort }}\right)$ was divided by the numerical density of nonsclerosed glomeruli in cortex $\left(\mathrm{N}_{\mathrm{VNSG}, \text { Cort }}\right)$. The former was estimated in the single glycolmethacrylate sections used to count nonsclerotic and sclerotic glomerular profiles, while the latter was estimated by dividing Nglom $_{\mathrm{NSG}}$ by cortical volume.

Cortical volume was estimated using the Cavalieri principle by counting the number of stereological test grid points overlying the cortical area in every tenth section used for point counting $(51,52)$. The following formula was used to calculate cortical volume: Cortical volume $=f_{1} \times f_{2} \times f_{3} \times \sum P \times a(p) \times T$, where $f_{1}$ is the weight fraction of tissue sampled, $f_{2}$ is the inverse of the slice sampling fraction, $f_{3}$ is the inverse of the section sampling fraction, $\Sigma P$ is the total number of grid points overlying cortex in sections, $a(p)$ is the area of the grid associated with each point, and $T$ is section thickness.

Histopathology. Samples for histopathological analysis were fixed in $10 \%$ formalin, embedded in paraffin, sectioned at 2-3 $\mu \mathrm{m}$, and stained with PAS. Glomerulosclerosis was determined using a standardized GSI 
Table 3. Total nephron number in normotensive subjects from 7 races

\begin{tabular}{lccc}
\hline Race & N & \multicolumn{1}{c}{ Nglom $_{\text {TOTAL }}$} & \multicolumn{1}{c}{ Ref. } \\
Japanese & 9 & $666,140 \pm 159,755$ & Present study \\
Danish & 37 & $617,000 \pm 154,000$ & 54 \\
Australian Aborigines & 17 & $683,174 \pm 130,220$ & 7 \\
Senegalese & 39 & $972,825 \pm 277,237$ & 55 \\
African American & 48 & $951,807 \pm 268,798$ & 14 \\
White American & 55 & $901,011 \pm 298,334$ & 14 \\
German & 10 & $1,402,360 \pm 346,357$ & 4 \\
In all studies, estimates were obtained using the physical disector/fractionator combination.
\end{tabular}

In all studies, estimates were obtained using the physical disector/fractionator combination.

(53). Briefly, the percentage of sclerotic glomeruli was estimated by counting sclerosed and nonsclerosed glomeruli in nonoverlapping microscopic fields $(\times 100)$ with at least 100 glomeruli counted per subject $(6)$.

Statistics. Statistical analyses were performed using Prism 7 (GraphPad Software) and Stata 13 (StataCorp). Values are the mean \pm SD unless otherwise stated. Differences between groups were analyzed using a KruskalWallis test with post-hoc Mann-Whitney $U$ tests with the Bonferroni correction. Measures of association were tested by Spearman rank coefficients. Statistical significance was defined as $P$ less than or equal to 0.05 .

Study approval. The study was approved by the Ethics Review Board of the Jikei University School of Medicine (approval 25-176, 7311), Nippon Medical School (approval 26-01), and the Human Research Ethics Committee of Monash University (approval 2016-0306), and subjects or family members provided written informed consent for autopsy.

\section{Author contributions}

JFB supervised and coordinated all aspects of this study. GK and VGP contributed equally to this work and designed the experiments, analyzed the data, and wrote the paper. Project planning was done by GK, VGP, LACM, WEH, NT, TY, and JFB. Counting work was performed by GK and LACM. Data analysis was performed by GK, VGP, LACM, MDH, KMD, and JFB. YO and AS provided clinical data and autopsy samples. AS and $\mathrm{MH}$ analyzed and interpreted the histological data. KMD provided renal physiological advice. NT and TY supervised clinical interpretation and provided funding. The manuscript was written by GK, VGP, WEH, NT, MDH, KMD, and JFB and the final version was approved by all authors.

\section{Acknowledgments}

This work was supported by the Japan Kidney Foundation Research Grant and JSPS KAKENHI grant numbers JP25461236 and JP16K0936 (to NT). VGP was awarded a CJ Martin Fellowship (NHMRC 1128582). The authors acknowledge the expert assistance of staff at the Monash Histology Platform who cut and stained the glycolmethacrylate sections. The funding sources played no role in study design, data collection, analysis, or interpretation; writing of the report; or the decision to submit the paper for publication.

Address correspondence to: John F. Bertram, Monash Biomedicine Discovery Institute and Department of Anatomy and Developmental Biology, Monash University, Clayton, Victoria, 3800, Australia. Phone: 61.3.9902.9101; Email: john.bertram@monash.edu.

1. Brenner BM, Garcia DL, Anderson S. Glomeruli and blood pressure. Less of one, more the other? Am J Hypertens. 1988;1(4 pt 1):335-347.

2. Puelles VG, Hoy WE, Hughson MD, Diouf B, Douglas-Denton RN, Bertram JF. Glomerular number and size variability and risk for kidney disease. Curr Opin Nephrol Hypertens. 2011;20(1):7-15.

3. Bertram JF, et al. Why and how we determine nephron number. Pediatr Nephrol. 2014;29(4):575-580.

4. Keller G, Zimmer G, Mall G, Ritz E, Amann K. Nephron number in patients with primary hypertension. $N$ Engl J Med. 2003;348(2):101-108.

5. Hughson MD, Douglas-Denton R, Bertram JF, Hoy WE. Hypertension, glomerular number, and birth weight in African Americans and white subjects in the southeastern United States. Kidney Int. 2006;69(4):671-678.

6. Hughson MD, Puelles VG, Hoy WE, Douglas-Denton RN, Mott SA, Bertram JF. Hypertension, glomerular hypertrophy and nephrosclerosis: the effect of race. Nephrol Dial Transplant. 2014;29(7):1399-1409.

7. Hoy WE, Hughson MD, Singh GR, Douglas-Denton R, Bertram JF. Reduced nephron number and glomerulomegaly in Aus- 
tralian Aborigines: a group at high risk for renal disease and hypertension. Kidney Int. 2006;70(1):104-110.

8. Liyanage T, et al. Worldwide access to treatment for end-stage kidney disease: a systematic review. Lancet. 2015;385(9981):1975-1982.

9. Tsuboi N, et al. Glomerular density in renal biopsy specimens predicts the long-term prognosis of IgA nephropathy. Clin $\mathrm{J}$ Am Soc Nephrol. 2010;5(1):39-44.

10. Tsuboi N, Kanzaki G, Koike K, Kawamura T, Ogura M, Yokoo T. Clinicopathological assessment of the nephron number. Clin Kidney J. 2014;7(2):107-114.

11. Kanzaki G, et al. Factors associated with a vicious cycle involving a low nephron number, hypertension and chronic kidney disease. Hypertens Res. 2015;38(10):633-641.

12. Iseki K. Chronic kidney disease in Japan from early predictions to current facts. Nephron Clin Pract. 2008;110(4):c268-c272.

13. Hoy WE, Douglas-Denton RN, Hughson MD, Cass A, Johnson K, Bertram JF. A stereological study of glomerular number and volume: preliminary findings in a multiracial study of kidneys at autopsy. Kidney Int Suppl. 2003;63(83):S31-S37.

14. Hughson MD, Gobe GC, Hoy WE, Manning RD, Douglas-Denton R, Bertram JF. Associations of glomerular number and birth weight with clinicopathological features of African Americans and whites. Am J Kidney Dis. 2008;52(1):18-28.

15. Hoy WE, Hughson MD, Bertram JF, Douglas-Denton R, Amann K. Nephron number, hypertension, renal disease, and renal failure. J Am Soc Nephrol. 2005;16(9):2557-2564.

16. Osterby R, Gundersen HJ. Glomerular size and structure in diabetes mellitus. I. Early abnormalities. Diabetologia. $1975 ; 11(3): 225-229$

17. Cachat F, Combescure C, Cauderay M, Girardin E, Chehade H. A systematic review of glomerular hyperfiltration assessment and definition in the medical literature. Clin J Am Soc Nephrol. 2015;10(3):382-389.

18. Helal I, Fick-Brosnahan GM, Reed-Gitomer B, Schrier RW. Glomerular hyperfiltration: definitions, mechanisms and clinical implications. Nat Rev Nephrol. 2012;8(5):293-300.

19. Lenihan CR, Busque S, Derby G, Blouch K, Myers BD, Tan JC. Longitudinal study of living kidney donor glomerular dynamics after nephrectomy. J Clin Invest. 2015;125(3):1311-1318.

20. Lenihan CR, Myers BD, Tan JC. Glomerular function and structure in living donors: lessons from single nephron studies. Curr Transplant Rep. 2016;3:24-32.

21. Tan JC, Workeneh B, Busque S, Blouch K, Derby G, Myers BD. Glomerular function, structure, and number in renal allografts from older deceased donors. J Am Soc Nephrol. 2009;20(1):181-188.

22. Denic A, et al. Single-nephron glomerular filtration rate in healthy adults. N Engl J Med. 2017;376(24):2349-2357.

23. Denic A, et al. The substantial loss of nephrons in healthy human kidneys with aging. J Am Soc Nephrol. 2017;28(1):313-320.

24. Zhang Z, et al. A common RET variant is associated with reduced newborn kidney size and function. J Am Soc Nephrol. 2008;19(10):2027-2034.

25. Quinlan J, et al. A common variant of the PAX2 gene is associated with reduced newborn kidney size. J Am Soc Nephrol. 2007;18(6):1915-1921.

26. Kagawa M, Tahara Y, Moji K, Nakao R, Aoyagi K, Hills AP. Secular changes in growth among Japanese children over 100 years (1900-2000). Asia Pac J Clin Nutr. 2011;20(2):180-189.

27. Luyckx VA, et al. Effect of fetal and child health on kidney development and long-term risk of hypertension and kidney disease Lancet. 2013;382(9888):273-283.

28. Luyckx VA, et al. A developmental approach to the prevention of hypertension and kidney disease: a report from the Low Birth Weight and Nephron Number Working Group. Lancet. 2017;390(10092):424-428.

29. Roseboom T, de Rooij S, Painter R. The Dutch famine and its long-term consequences for adult health. Early Hum Dev. 2006;82(8):485-491.

30. Stanner SA, et al. Does malnutrition in utero determine diabetes and coronary heart disease in adulthood? Results from the Leningrad siege study, a cross sectional study. BMJ. 1997;315(7119):1342-1348.

31. Moritz KM, Singh RR, Probyn ME, Denton KM. Developmental programming of a reduced nephron endowment: more than just a baby's birth weight. Am J Physiol Renal Physiol. 2009;296(1):F1-F9.

32. Hoy WE, Ingelfinger JR, Hallan S, Hughson MD, Mott SA, Bertram JF. The early development of the kidney and implications for future health. J Dev Orig Health Dis. 2010;1(4):216-233.

33. Luyckx VA, Brenner BM. Birth weight, malnutrition and kidney-associated outcomes - a global concern. Nat Rev Nephrol. 2015;11(3):135-149.

34. Hayman J, Martin JW, Miller M. Renal function and the number of glomeruli in the human kidney. Arch Intern Med. 1939;64(1):69-83

35. Johnson RJ, Feig DI, Nakagawa T, Sanchez-Lozada LG, Rodriguez-Iturbe B. Pathogenesis of essential hypertension: historical paradigms and modern insights. J Hypertens. 2008;26(3):381-391.

36. Glassock RJ, Rule AD. The implications of anatomical and functional changes of the aging kidney: with an emphasis on the glomeruli. Kidney Int. 2012;82(3):270-277.

37. Fields LE, Burt VL, Cutler JA, Hughes J, Roccella EJ, Sorlie P. The burden of adult hypertension in the United States 1999 to 2000: a rising tide. Hypertension. 2004;44(4):398-404.

38. Miura K, Nagai M, Ohkubo T. Epidemiology of hypertension in Japan: where are we now? Circ J. 2013;77(9):2226-2231.

39. Denic A, et al. Detection and clinical patterns of nephron hypertrophy and nephrosclerosis among apparently healthy adults. Am J Kidney Dis. 2016;68(1):58-67.

40. Hughson MD, et al. APOL1 risk alleles are associated with more severe arteriosclerosis in renal resistance vessels with aging and hypertension. Kidney Int Rep. 2016;1(1):10-23.

41. Kopp JB. Rethinking hypertensive kidney disease: arterionephrosclerosis as a genetic, metabolic, and inflammatory disorder Curr Opin Nephrol Hypertens. 2013;22(3):266-272.

42. Carlström M, Wilcox CS, Arendshorst WJ. Renal autoregulation in health and disease. Physiol Rev. 2015;95(2):405-511.

43. Elsherbiny HE, et al. Nephron hypertrophy and glomerulosclerosis and their association with kidney function and risk factors among living kidney donors. Clin J Am Soc Nephrol. 2014;9(11):1892-1902.

44. Vallon V, Blantz RC, Thomson S. Glomerular hyperfiltration and the salt paradox in early [corrected] type 1 diabetes mellitus: a 
tubulo-centric view. J Am Soc Nephrol. 2003;14(2):530-537.

45. Rosenberg AZ, et al. The application of digital pathology to improve accuracy in glomerular enumeration in renal biopsies. PLoS One. 2016;11(6):e0156441.

46. Matsuo S, et al. Revised equations for estimated GFR from serum creatinine in Japan. Am J Kidney Dis. 2009;53(6):982-992.

47. Fujimoto S, Watanabe T, Sakamoto A, Yukawa K, Morimoto K. [Studies on the physical surface area of Japanese. 18. Calculation formulas in three stages over all ages]. Nihon Eiseigaku Zasshi. 1968;23(5):443-450.

48. Bertram JF. Counting in the kidney. Kidney Int. 2001;59(2):792-796.

49. Cullen-McEwen LA, Douglas-Denton RN, Bertram JF. Estimating total nephron number in the adult kidney using the physical disector/fractionator combination. Methods Mol Biol. 2012;886:333-350.

50. Gundersen HJ. Estimators of the number of objects per area unbiased by edge effects. Microsc Acta. 1978;81(2):107-117.

51. Nyengaard JR. Stereologic methods and their application in kidney research. J Am Soc Nephrol. 1999;10(5):1100-1123.

52. Bertram JF. Analyzing renal glomeruli with the new stereology. Int Rev Cytol. 1995;161:111-172.

53. Changsirikulchai S, et al. Platelet-derived growth factor-D expression in developing and mature human kidneys. Kidney Int. 2002;62(6):2043-2054

54. Nyengaard JR, Bendtsen TF. Glomerular number and size in relation to age, kidney weight, and body surface in normal man. Anat Rec. 1992;232(2):194-201.

55. McNamara BJ, Diouf B, Douglas-Denton RN, Hughson MD, Hoy WE, Bertram JF. A comparison of nephron number, glomerular volume and kidney weight in Senegalese Africans and African Americans. Nephrol Dial Transplant. 2010;25(5):1514-1520. 